\title{
ЕЩЕ ОБ ОДНОМ СПОСОБЕ УСКОРЕНИЯ СХОДИМОСТИ ПРОЦЕССА АДАПТАЦИИ
}

\author{
Клименко А.К., канд. техн. наук, доцент \\ Бердянский государственный педагогический университет
}

\begin{abstract}
Аннотация: Сущзествуют системы адаптивного управления цииклиески повторяющимися технологическими процессами. Управляющая программа корректируется на основании ошибок в предшествующих цииклах её воспроизведения. Для оптимизации процесса адаптации в условиях помех требуются дополнительные проигрывания управляющей программы. Предлагается ускорение прочесса адаптации посредством исключения дополнительных проигрываний.

Annotation: There are systems of adaptive control of cyclically repeating technological processes. The controlling program is corrected on the base of errors in the previous cycles of its execution. For optimisation of the process of adaptation in the conditions of noise the additional executions of the controlling program are required. Acceleration of process of adaptation by the method of exception of additional executions is offered.
\end{abstract}

Ключевые слова: управляющая программа, автоматизированный технологический объект, дискретный интегратор, обратная модель, средний квадрат ошибки, блок статистического анализа.

Введение. В настоящее время возникают проблемы при создании адаптивных систем управления. Существует различная терминология в области этих систем. В числе адаптивных называются самонастраивающиеся, самоорганизующиеся, самообучающиеся и обучаемые системы. В качестве обучаемых могут выступать системы программного управления циклически повторяющимися технологическими процессами с адаптивной корректировкой управляющих программ. Важным показателем качества обучаемых систем является скорость сходимости процесса адаптации в условиях случайных возмущающих воздействий. Имеются технические решения по ускорению процесса адаптации. В данной работе рассматриваются еще один способ ускорения процесса адаптации, более эффективный в сравнении с известным.

Анализ известных источников. Известны автоматизированные технологические объекты (АТО), в которых управление осуществляется по циклически повторяющимся программам. Примерами таких АТО являются металлорежущие станки с программным управлением и станы горячей прокатки. Управляющие программы готовятся в условиях неполной информации об объекте управления и возмущающих воздействиях. Поэтому требуется корректировка управляющих программ как во время их разработки, так и в процессе эксплуатации. Системы, в которых осуществляется такая корректировка, называются системами с изменяемой программой (1), системами с самоустанавливающейся программой [2], самонастраивающимися системами «от детали к детали» [3,4] и адаптивными системами [5]. Далее их будем называть системами с адаптивной корректировкой управляющих программ или обучаемыми системами.

Классическая структурная схема адаптивной системы показана на рис.1. Символами на схеме обозначены: УП управляющая программа, АТО - автоматизированный технологический объект, ОМ - обратная модель, ДИ дискретный интегратор, БСА - блок статистического анализа. Знаком умножения обозначено безынерционное звено с изменяемым коэффициентом усиления $\gamma_{v}$.

Работа системы состоит в следующем. Предполагается, что АТО является односвязным непрерывным объектом, но все приведенные на схеме сигналы рассматриваются в дискретном времени (являются решетчатыми функциями). Управляющая программа $x(n)$ циклически повторяется и не изменяется от цикла к циклу. Она же служит и для измерения ошибок обработанных деталей. Продолжительность программы $-N$ тактов дискретного времени. Ошибки могут быть измерены как одновременно с выполнением программы (на прокатном стане), так и в промежутках между циклами (металлорежущие станки). АТО является непрерывным, но его выходной сигнал оценивается в дискретном времени:

$$
y(n+\Gamma), 0 \leq \gamma \leq 1, n=0,1,2, \ldots, N
$$

где $n$ - дискретное время, $\gamma$ - дробная часть дискретного времени.

Ошибка каждого цикла воспроизведения программы измеряется в промежутках между циклами и регистрируется в буферной памяти. Регистрируется только ее значения в привязке к дискретным моментам времени:

$$
\varepsilon_{k}(n)=x(n)-y_{k}(n), n \in[0, N],
$$

где $k$ - номер цикла воспроизведения программы. 


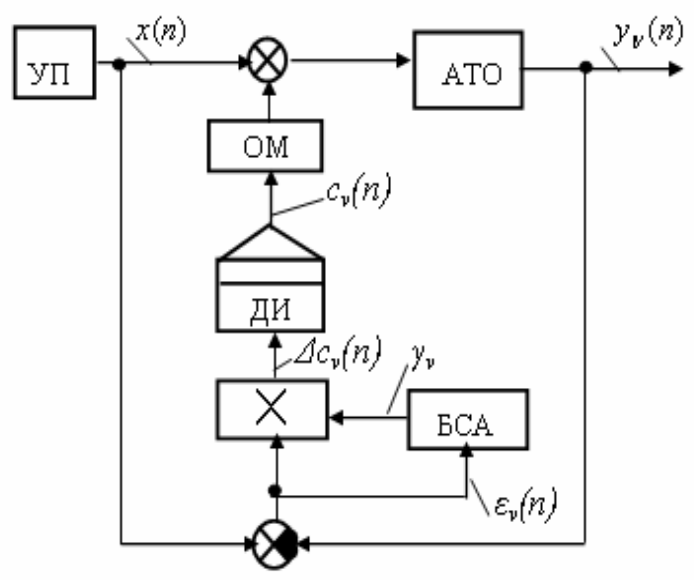

Рис.1 - Структурная схема адаптивной системы.

На основании зарегистрированной ошибки формируется поправка на программу Д $c_{v}(n)$, которая подается на вход ДИ.

Корректировка программ в общем случае осуществляется по формуле

$$
c_{v}(n)=c_{v-1}(n)+Д c_{v}(n)=c_{v-1}(n)+\gamma_{v} f\left\{\varepsilon_{v-1}(n)\right\}, n \in[0, N], v=1,2, \ldots,
$$

где $\boldsymbol{v}$-номер цикла корректировки, $c_{v}(n)$ - корректирующий сигнал, $\gamma_{v}$ - коэффициент усиления в контуре адаптивной корректировки, $\varepsilon_{v-1}(n)$ - ошибка предшествующего цикла воспроизведения управляющей программы, $f\{\cdot\}$ - функция, определяемая этой ошибкой и обеспечивающая сходимость процесса ее минимизации.

Ошибки изготовления деталей состоят из регулярной и случайной составляющих. Последняя является центрированной аддитивной. Источником ее могут выступать как процесс обработки деталей сложной формы, так и ошибки автоматического измерения размеров последних. Целью адаптации является формирование корректирующего сигнала, обеспечивающего сведение к нулю регулярной составляющей ошибки. Для этого требуется сходимость процесса (1). В опубликованных работах рассматривались задачи обеспечения сходимости процессов как при отсутствии случайной составляющей ошибки, так и при наличии ее.

Как было показано Андрейчиковым Б.И. [4], при реализации контура адаптации на аналоговых средствах автоматики и вычислительной техники процесс адаптации сходится на первых циклах воспроизведения программ и неизбежно расходится в последующих.

В системах с квантованным по времени представлением информации имеются технические решения по обеспечению сходимости в условиях отсутствия помех, но и они обладают существенными недостатками. В работе [6] предложена адаптивная система, в которой в качестве формирователя корректирующего сигнала используется безынерционное динамическое звено. В этом техническом решении имеет место низкая скорость процесса адаптации.

Попытки обеспечения сходимости процесса адаптации в условиях помех были менее успешными. Так, например, в работе [7] показано, что при корректировке управляющей программы по формуле (1) в условиях случайных помех величина коэффициента усиления $\Gamma_{н}(n)$ зависит от параметров помех. Для определения оптимального коэффициента усиления требуется трудоемкий статистический анализ объекта управления, что на практике нерентабельно.

В решении задачи обеспечения сходимости процесса адаптации в условиях помех оказалось эффективным применение математического аппарата - метода стохастической аппроксимации. Рекомендации по его использованию даны в [5]. Проблемы обеспечения сходимости порождаются тем обстоятельством, что условия сходимости для регулярной и случайной составляющих ошибки различны. Как следует из [5], для обеспечения сходимости процесса (1) в условиях, когда регулярная и случайная составляющие возмущающего воздействия стационарны, коэффициент усиления в контуре адаптации должен удовлетворять требованиям:

$$
\sum_{v=1}^{\infty} \gamma_{v}=\infty,(2)
$$




$$
\sum_{v=1}^{\infty} \gamma_{v}^{2}<\infty
$$

Условие (2) относится к регулярной составляющей ошибки, а условие (3) - к случайной составляющей. Выполнение последнего требует бесконечного числа циклов корректировки с уменьшением в каждом цикле коэффициента усиления со стремлением его к нулю.

Основополагающим техническим решением в задачах обеспечения сходимости процесса адаптации, как для регулярной, так и случайной составляющих ошибки, явилось использование в адаптивном контуре системы обратной модели (ОМ) АТО.

В работе [8] показано, что в случае отсутствия помех можно обеспечить полную корректировку программы уже после одного цикла ее воспроизведения. Для этого необходимо осуществить ОМ АТО на средствах дискретной вычислительной техники и обеспечить ввод корректирующего сигнала с упреждением на один такт дискретного времени. Указанная ОМ должна удовлетворять следующему требованию: переходная характеристика комплекса из последовательно соединенных ОМ и АТО в дискретном времени имеет вид:

$$
h_{k}(n)=\left\{\begin{array}{l}
1 \text { nри } n \geq 1, \\
0 \text { nри } n \leq 0,
\end{array}\right.
$$

где $n$ - дискретное время.

Техническое решение дискретной ОМ, удовлетворяющей условию (4), было найдено на базе компьютерных технологий. Оно защищено авторским свидетельством [9].

Далее для общей ошибки $v$-го цикла воспроизведения программы принимается выражение:

$$
\varepsilon_{v}(n)=\bar{\varepsilon}_{v}(n)+\widetilde{\varepsilon}_{v}(n), n \in[0, N](5)
$$

где $\bar{\varepsilon}_{v}(n)$ и $\widetilde{\varepsilon}_{v}(n)$ - соответственно регулярная и случайная составляющие.

При решении задачи сходимости процесса адаптации в качестве статистических характеристик регулярной и случайной составляющих зарегистрированных ошибок принимаются их среднеквадратичные значения на интервале цикла $N$. Поскольку случайная составляющая принимается аддитивной, между средним квадратом общей ошибки и средними квадратами ее составляющих справедливо соотношение:

$$
M\left\{\left[\varepsilon_{v}(n)\right]^{2}\right\}=M\left\{\left[\bar{\varepsilon}_{v}(n)\right]^{2}\right\}+M\left\{\left[\widetilde{\varepsilon}_{v}(n)\right]^{2}\right\}, n \in[0, N] \text {. (6) }
$$

В работе [10] найдено условие оптимальности коэффициента усиления $\gamma_{v}$ в процессе (1) для обеспечения минимума среднего квадрата ошибки управляющей программы после ее корректировки. Это условие определяется следующим соотношением между регулярной и случайной составляющими зарегистрированной ошибки воспроизведения программы:

$$
\Gamma_{\mathrm{H}+1}=\frac{M\left\{\left[\overline{\mathrm{e}}_{\mathrm{H}}(n)\right]^{2}\right\}}{M\left\{\left[\mathrm{e}_{\mathrm{H}}(n)\right]^{2}\right\}+M\left\{\left[\tilde{\mathrm{e}}_{\mathrm{H}}(n)\right]^{2}\right\}} . \text { (7) }
$$

В этой же работе указывается, что оптимальное значение коэффициента усиления, описываемое выражением (7), может быть определено и при отсутствии возможности измерения регулярной и случайной составляющих ошибки. Это достигается за счет двухкратного воспроизведения управляющей программы перед ее корректировкой.

Важным показателем качества адаптивной системы является высокая скорость сходимости процесса (1). Оптимизация коэффициента усиления по формуле (7) ускоряет процесс сходимости процесса адаптации при работе адаптивной системы в условиях случайных возмущающих воздействий. Но это ускорение является недостаточным. Необходимость двухкратного воспроизведения управляющей программы для осуществления одного шага ее корректировки в два раза снижает скорость сходимости.

Требуется поиск технических решений по устранению этого недостатка.

В работе [11] предложен способ повышения скорости сходимости процесса адаптации в системе по рис.1 с двухкратным воспроизведением управляющей программы. Структурная схема этой системы аналогична изображенной на рис.1, но для ускорения сходимости при формировании корректирующего сигнала используется среднее значение ошибок двух циклов воспроизведения программы. В этом случае оптимальный коэффициент усиления адаптивного контура определяется выражением:

$$
\Gamma_{v+1}=\frac{2 M\left\{\left[\overline{\mathrm{e}}_{\mathrm{H}}(n)\right]^{2}\right\}}{2 M\left\{\left[\overline{\mathrm{e}}_{\mathrm{H}}(n)\right]^{2}\right\}+M\left\{\left[\tilde{\mathrm{e}}_{\mathrm{H}}(n)\right]^{2}\right\}} .
$$

Реализация адаптивной системы, в которой коэффициент усиления адаптивного контура определяется условием (8), дает некоторое ускорение процесса, но оно лишь частично компенсирует потерю скорости из-за двухкратного воспроизведения управляющей программы. 
Целью данной статьи является устранения указанного недостатка. Предполагается такое осуществление адаптивного контура системы, которое обеспечивает возможность адаптивной корректировки управляющей программы в каждом цикле ее воспроизведения с выполнением условия оптимальности (7). Необходимость введения вспомогательных циклов воспроизведения программы при этом устраняется, что ускоряет процесс адаптации.

Исходные данные и постановка задачи. Рассматривается вопрос ускорения сходимости процесса адаптации в системе, описанной в работе [10]. Структурная схема адаптивной системе изображена на рис.1.

Входящая в систему ОМ выполнена таким образом, что импульсная переходная функция комплекса «ОМ-АТО» удовлетворяет требованиям (4). ОМ отличается от идеальной временным запаздыванием на один такт дискретного времени. Поэтому зарегистрированный сигнал ошибки подается но вход ДИ с опережающим временным сдвигом на ту же величину, т.е.

$$
\text { Д } c_{v}(n)=\gamma_{v} \varepsilon_{v}(n+1) .(9)
$$

Будем предполагать, что в системе имеют место случайные возмущающие воздействия, которые приложены к точке измерения ошибки воспроизведения программы. Возмущающие воздействия являются аддитивными. Поэтому составляющие ошибок соседних циклов воспроизведения программы удовлетворяют требованиям:

$$
\bar{\varepsilon}_{v}(n)=\bar{\varepsilon}_{v-1}(n), \quad \widetilde{\varepsilon}_{v}(n) \neq \widetilde{\varepsilon}_{v-1}(n), M\left\{\left[\widetilde{\varepsilon}_{v}(n)\right]^{2}\right\}=M\left\{\left[\widetilde{\varepsilon}_{v-1}(n)\right]^{2}\right\} .(10)
$$

Критерием оптимальности процесса адаптации является стремление к нулю среднего квадрата регулярной составляющей ошибки на всем интервале программы, т.е.

$$
\lim _{v \rightarrow \infty} M\left\{\left[\bar{\varepsilon}_{v}(n)\right]^{2}\right\}=0, n \in[0, N] .
$$

БСА предназначен для нахождения оптимального $\gamma_{v+1}$, обеспечивающего минимум среднеквадратичной ошибки воспроизведения программы после ее корректировки по результатам анализа ошибок предыдущих воспроизведений в соответствии с условием (7).

Задачей данной работы является ускорение процесса адаптации в адаптивной системе по рис.1 посредством исключения из алгоритма ее работы вспомогательных проигрываний управляющей программы в промежутках между рабочими циклами воспроизведения. Эта задача решается разработкой такого БСА, входящего в адаптивную систему, для функционирования которого достаточна информация об ошибках воспроизведения управляющей программы в предшествующих рабочих циклах. БСА должен обеспечить условие оптимальности (7). Требуется найти математическое описание вновь созданного БСА и предложить его структурную схему.

Решение задачи. Для упрощения задачи создания БСА с указанными требованиями преобразуем запись условия оптимальности (7). Из соотношения (6) следует, что для регулярной составляющей ошибки справедливо выражение:

$$
M\left\{\left[\bar{\varepsilon}_{v}(n)\right]^{2}\right\}=M\left\{\left[\varepsilon_{v}(n)\right]^{2}\right\}-M\left\{\left[\widetilde{\varepsilon}_{v}(n)\right]^{2}\right\} .
$$

Подставляя полученное выражение в соотношение (7), после преобразований получим формулу для оптимального коэффициента усиления в адаптивном контуре:

$$
\gamma_{v+1}=1-\frac{M\left\{\left[\tilde{\varepsilon}_{v}(n)\right]^{2}\right\}}{M\left\{\left[\varepsilon_{v}(n)\right]^{2}\right\}} .
$$

Сначала рассмотрим задачу определения численных значений входящих в выражение (11) средних квадратов при работе адаптивной системы по рис.1. Эта задача выполняется с помощью блока статистического анализа БСА, структурная схема которого изображена на рис2. В состав БСА входят буферная память, элемент задержки, сумматор, блок умножения и вычислитель. БСА функционирует следующим образом.

Регистрируются ошибки двух предшествующих циклов воспроизведения программы. В промежутках между циклами в ДИ вносится поправка в соответствии с выражением (9). Ошибка до корректировки имеет вид

а после корректировки преобразуется к виду

$$
\varepsilon_{v-1}(n)=\bar{\varepsilon}_{v-1}(n)+\widetilde{\varepsilon}_{v-1}(n),(12)
$$

$$
\varepsilon_{v}(n)=\left(1-\gamma_{v}\right) \varepsilon_{V-1}(n)+\widetilde{\varepsilon}_{v}(n),(13)
$$

где $\gamma_{v}$ - коэффициент усиления в адаптивном контуре при $v$-м цикле воспроизведения программы. 


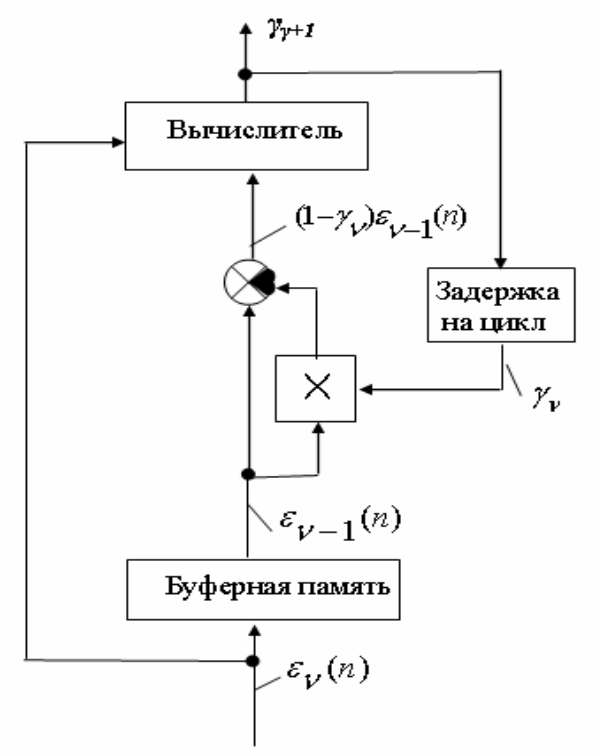

Рис. 2 - Структурная схема блока статистического анализа

В БСА формируется следующая разность между зарегистрированными ошибками:

$$
\Delta \varepsilon=\varepsilon_{v}(n)-\left(1-\gamma_{v}\right) \varepsilon_{v-1}(n),
$$

которая после преобразований с учетом соотношений $(10,12,13)$ получает вид:

$$
\Delta \varepsilon=\widetilde{\varepsilon}_{v}(n)-\widetilde{\varepsilon}_{v-1}(n) \text {. }
$$

Находим средний квадрат полученной разности с учетом аддитивности помехи:

$$
M\left\{[Д \varepsilon]^{2}\right\}=M\left\{\left[\varepsilon_{v}(n)-\left(1-\gamma_{v}\right) \varepsilon_{v-1}(n)\right]^{2}\right\}=M\left\{\left[\widetilde{\varepsilon}_{v}(n)\right]^{2}\right\}+M\left\{\left[\widetilde{\varepsilon}_{v-1}(n)\right]^{2}\right\}=2 M\left\{\left[\widetilde{\varepsilon}_{v}(n)\right]^{2} .\right.
$$

Из полученной зависимости находим выражение для среднего квадрата случайной составляющей ошибки при $v$ - м воспроизведении программы:

$$
M\left\{\left[\tilde{\varepsilon}_{v}(n)\right]^{2}\right\}=\frac{M\left\{\left[\varepsilon_{v}(n)-\left(1-\gamma_{v}\right) \varepsilon_{v-1}(n)\right]^{2}\right\}}{2} .
$$

Подставив полученное значение среднего квадрата случайной составляющей ошибки в формулу (11), находим математическое описание для искомого БСА, структурная схема которого изображена на рис.2:

$$
\gamma_{v+1}=1-\frac{M\left\{\left[\varepsilon_{v}(n)-\left(1-\gamma_{v}\right) \varepsilon_{v-1}(n)\right]^{2}\right\}}{2 M\left\{\left[\varepsilon_{v}(n)\right]^{2}\right\}} .
$$

Реализация БСА с использованием в его вычислителе формулы (14) дает возможность находить оптимальный коэффициент усиления без промежуточных вспомогательных воспроизведений программы. При этом необходимо иметь информацию о зарегистрированных ошибках двух предшествующих циклов воспроизведения программы, а также информацию о коэффициенте усиления $\gamma_{v}$, использованном при корректировке программы в $v$ - м цикле. Если же указанная корректировка не производилась, то в формуле (14) необходимо принять коэффициент усиления предыдущего цикла воспроизведения программы равным нулю $\left(\gamma_{v}=0\right)$.

Экспериментальная часть. Полученные результаты подтверждены моделированием. Из анализа формул (11) и (14) следует, что величина получаемого коэффициента усиления может изменяться в пределах от 0 до 1 в зависимости от соотношения случайной составляющей ошибки к регулярной. Например, при одинаковой интенсивности регулярной и случайной составляющих, когда $M\left\{\left[\bar{\varepsilon}_{v}(n)\right]^{2}\right\}=M\left\{\left[\widetilde{\varepsilon}_{v}(n)\right]^{2}\right\} \neq 0$, оптимальный коэффициент усиления равен $1 / 2$. Если в зарегистрированных ошибках управления присутствует только случайная составляющая (т.е. $M\left\{\left[\tilde{\varepsilon}_{v}(n)\right]^{2}\right\} \neq 0$ при $M\left\{\left[\bar{\varepsilon}_{v}(n)\right]^{2}\right\}=0$ ), то вычисленный коэффициент усиления равен нулю, а процесс адаптации заканчивается без внесения поправки в дискретный интегратор. В случае же присутствия только регулярной составляющей, т.е. при $M\left\{\left[\widetilde{\varepsilon}_{v}(n)\right]^{2}\right\}=0$, коэффициент усиления равен единице и процесс адаптации 
заканчивается после единственного внесения в дискретный интегратор значения зарегистрированной ошибки $\varepsilon_{v}(n)$. Такие ситуации могут возникать как при первых циклах воспроизведения программы, так и при любых последующих.

Выводы. Использование в адаптивной системе ОМ АТО совместно с предлагаемым способом подстройки коэффициента усиления обеспечивает оптимальную корректировку программы в любых циклах ее воспроизведения. Возмущающие воздействия при этом могут быть как стационарными, так и медленно изменяющимися во времени. Для получения статистических данных об ошибках не требуется проведения экспериментальных проигрываний программы, что вдвое ускоряет процесс адаптации.

\section{Литература}

1. Ивахненко А.Г. Техническая кибернетика / А.Г. Ивахненко. - Киев: Гостехиздат УССР, 1959. - 423 с.

2. Перельман И.И. Регулирование по принципу самоустанавливающейся программы / И.И. Перельман // Автоматика и телемеханика. - 1958, №9. - С. 813-823.

3. Кобринский А.Е. Самонастраивающаяся система программного управления станками / А.Е. Кобринский, А.Ш. Колискор, Е.И. Левковский [и др.] // Вестник АН СССР. - 1965, №9. - С. 52-57.

4. Андрейчиков Б.И. Динамическая точность систем программного управления станками / Б.И. Андрейчиков. - М.: Машиностроение, 1964. - С. 242-267.

5. Цыпкин Я.З. Адаптация и обучение в автоматических системах / Я.З Цыпкин. - М.: Наука, 1968.400 с.

6. Перельман И.И. Самонастраивающаяся программа как средство для получения заданной реакции на выходе линейного динамического объекта регулирования / И.И. Перельман // Автоматика и телемеханика. - 1958. №4. - С.312-320.

7. Перельман И.И. Выбор оптимального коэффициента усиления в системе регулирования по самоустанавливающейся программе / И.И. Перельман // Автоматика и телемеханика. -1959, №2. - С.184191.

8. Клименко А.К. О сходимости процессов алаптации в цифровых системах программного управления станками / А.К. Клименко // В сб. «Адаптивные системы управления металлорежущими станками» под ред. А.Е. Кобринского, Сер. С-1. - М.: НИИМАШ, 1971.- С.58-67

9. А. с. СССР 1406563 , МКИ G 05 В 5/01. Корректирующее устройство / Клименко А.К., Клименко В.Г. (СССР). - № 4041877/24-24; заявл. 25.03.86; опубл. 30.06.88, Бюл. №24.

10. Клименко А.К. Об оптимизации коэффициента усиления в адаптивной системе с обратной моделью / А.К. Клименко // Автоматика. Автоматизация. Электротехнические комплексы и системы. - 2006, №2. - С.125131.

11. Клименко А.К. Об ускорении сходимости процессов в адаптивной системе с обратной моделью / А.К. Клименко //Автоматика. Автоматизация. Электротехнические комплексы и системы. - 2007, №2. - С.140146. 